\title{
Chapter 4 \\ Three Simple Questions Not Related to Electric Fields
}

As a thought-provoking pastime for readers, the authors here offer three simple questions in technology or physics. No answers to these questions are given in this book.

\section{Question 1: Active Filter}

An active filter is also sometimes called active noise control. It is a method of eliminating unwanted or harmful noise by emitting sound with the same amplitude but the opposite phase, thereby cancelling out the original noise. However, the noise or sound has the energy of an acoustic wave.

Where has this sound energy gone when the noise is cancelled out?

\section{Question 2: Wind Turbine}

We frequently see large wind turbines for wind power generation. These turbines usually have two or three blades, much fewer than the vanes used in water turbines for hydraulic power generation.

Why is the number of blades in wind turbines so small? Don't turbines fail to catch the energy of the wind that passes between the blades?

\section{Question 3: Superconducting Coil}

Current keeps flowing at the same value as its initial state in a superconducting coil. This is the principle of SMES (Superconducting Magnetic Energy Storage) systems in which no ohmic losses occur because of the coil's zero resistance. However, it is well known that electrons in circular motion lose energy by radiating electromagnetic waves. Why can the current keep flowing in a superconducting coil? 